\title{
Comparison of Liquid-Based Cytology and Conventional Pap Smear Concerning Detectability of Cervicovaginal Infectious Agents
}

\author{
Besim Haluk Bacanakgil, @ Işık Kaban, ๑ Sezgi Güllü Erciyestepe
}

Deparment of Obstetrics and Gynaecology, Istanbul Training and Research Hospital, Istanbul, Turkey

\begin{abstract}
Introduction: The present study aims to compare Conventional Pap smear (CPS) and Liquid-based Cytology (LBC) results concerning detectability of cervicovaginal infectious agents.

Methods: The results of cervical cancer screening tests performed between 2005 and 2017 in a tertiary center in Istanbul were analyzed retrospectively. For this study, bacterial vaginosis, Trichomonas vaginalis, Candida, Actinomyces and Herpes simplex virus were evaluated as infectious agents of cervicovaginal infection.

Results: Smear results of 60014 women between the ages of 21-65 were examined. CPS technique was used in 58979 of these, and the LBC technique in 1035. Infectious agent was detected in $8.6 \%$ of CPS and $8 \%$ of LBC ( $p=0.503)$. Bacterial vaginosis was detected in 3.459 (5.9\%) women using CPC technique and $43(4.1 \%)$ women using LBC technique $(p=0.003)$. While Trichomonas vaginalis, Actinomyces and Herpes simplex in CPS technique were detected in $249(0.4 \%), 62(0.1 \%)$ and four (0.0\%) women, respectively, these agents were not seen with $L B C(p=0.034 ; p=0.627 ; p=N S$, respectively). Candida was detected in 1.342 (2.3\%) and 40 (3.9\%) women in CPS and LBC techniques, respectively $(p<0.001)$.

Discussion and Conclusion: According to the results of this study, the detectability of infectious agents, such as bacterial vaginosis, trichomonas vaginalis, and actinomyces, is lower in smear tests performed using the LBC technique compared to the CPS technique. Candida infections can be detected with the LBC technique. Clinicians can consider the advantages of the CPS technique in detecting cervicovaginal infectious agents.
\end{abstract}

Keywords: Cervix; cytology; pap; screening; smear.

P apanicolaou (Pap) cervical smear has been involved in gynecology practice for more than fifty years. In time, the "liquid-based" (such as SurePath, ThinPrep) methods of this conventional method have been developed and implemented. Pap smear terminology in 1994 was combined with the Bethesda system and this was revised in 2001. Ac- cording to the Bethesda system, five microorganisms have been identified in smears: Trichomonas vaginalis, Candida species, bacterial species (bacterial vaginosis), Actinomyces species and Herpes simplex virus ${ }^{[1]}$.

This study aimed to analyze infectious agents observed in conventional and liquid-based Pap cervical smears screening. 


\section{Materials and Methods}

Between 2005 and 2017, Pap smear pathology reports for screening in women aged 21-65 years were evaluated retrospectively. Sixty thousand fourteen smears, which were accepted as adequate from 72230 smears, were included in this study. Pap smears were examined by pathologists in two ways as follows: classical-conventional (CPS) and liquid-based cytology (LBC). ThinPrep 2000 (Cytyc Corp., MA, USA) was used in liquid-based cytological methods.

Data were analysed using the SPSS version 22.0. Fisher's exact test was used to compare the proportions of infectious agents identificated in the Pap smear reports. A p-value of $<0.05$ was considered statistically significant.

\section{Results}

The smear results of 60014 women were evaluated in this study. The mean age was $44.68 \pm 10.49 .58979$ (98.3\%) of the smears were conventional and 1035 (1.7\%) were liquidbased. Overall prevalence, 5199 (8.66\%) smear infectious organisms were detected. The prevalence of infectious organism was $8.6 \%$ in CPS and $8 \%$ in LBC. In this respect, no statistically significant difference was found between CPS and LBC $(p=0.503)$. Bacterial vaginosis was detected in 3.459 (5.9\%) women by CPC technique and 43 (4.1\%) women by LBC technique $(p=0.003)$. While Trichomonas vaginalis, Actinomyces and Herpes simplex in CPS technique were detected in $249(0.4 \%), 62(0.1 \%)$ and four $(0.0 \%)$ women, respectively, these agents were not seen with LBC ( $p=0.034 ; p=0.627 ; p=N S$, respectively). Candida was detected in $1.342(2.3 \%)$ and 40 (3.9\%) women in CPS and LBC techniques, respectively $(p<0.001)$ (Table 1$)$.

\section{Discussion}

In this study, we analyzed infectious agents in smear results obtained with CPS and LBC techniques. The overall rate of infectious organisms in cervical smears of our study cohort

Table 1. Infectious organisms in Pap smears

\begin{tabular}{lccc} 
& CPS $(\mathbf{n = 5 8 9 7 9 )}$ & $\mathbf{L B C}(\mathbf{n = 1 0 3 5 )}$ & $\mathbf{p}$ \\
& $\mathbf{n}(\mathbf{\%})$ & $\mathbf{n}(\mathbf{\%})$ & \\
\hline Infectious organisms & $5.116(8.6)$ & $83(8.0)$ & 0.503 \\
Bacterial vaginosis & $3.459(5.9)$ & $43(4.1)$ & 0.003 \\
Candida & $1.342(2.3)$ & $40(3.9)$ & 0.000 \\
Trichomonas vaginalis & $249(0.4)$ & 0 & 0.034 \\
Actinomyces & $62(0.1)$ & 0 & 0.627 \\
Herpes simplex & 4 & 0 & \\
\hline
\end{tabular}

${ }^{*}$ CPS: Conventional Pap smear; LBC: Liquid-based cytology (ThinPrep). is $8.66 \%$. The overall prevalence in studies with similar high cases in the literature ranges from $7.28 \%$ to $9.01 \%{ }^{[2-4]}$.

In this study, we detected an infectious organism in $8.6 \%$ of conventional smears and $8 \%$ of liquid-based smears. We found no statistically significant difference between conventional and LBC concerning overall infection rate. In the literature, the prevalence of infectious organisms is reported in the range of $8.45 \%-9.1 \%$ in conventional smears and $5.90 \%-9.50 \%$ in liquid-based smears ${ }^{[2-4]}$. On the other hand, in the study of Al-Awadhi et al. ${ }^{[2]}$, a significant difference was found in favor of the conventional method. In their study, a significant decrease was observed in the detection of organisms with the transition from the conventional method to the liquid-based method.

In our study, Bacterial vaginosis was higher in conventional smears than in liquid-based ones $(p<0.05)$, which is consistent with Takei et al.'s ${ }^{[1]}$ findings. Similarly, Trichomonas vaginalis was also higher in conventional smears than fluid-based $(p<0.05)$. This result is consistent with the studies of Takei et al. ${ }^{[1]}$ and Al-Awadhi ${ }^{[2]}$. The partial elimination of bacteria and Trichomonas in the technical process may explain less detection in the liquid-based method ${ }^{[1,2]}$.

In contrast, Candida was more prevalent in the liquid-based method $(p<0.05)$. Similarly, Candidiasis was significantly higher in liquid-based smears in Takei's study ${ }^{[1]}$. Because Candida species is larger than bacteria and Trichomonas organisms, they may not be eliminated in the liquid-based process and thus may be more intense in the sediment, and this may explain its more detection than in the conventional technique $^{[1]}$.

In conclusion, conventional Pap smear is more effective than a liquid-based technique for the detection of bacterial vaginosis and Trichomonas vaginalis. The LBC (ThinPrep) is more effective for candidiasis. The identification of gynecological infectious organisms in the Pap smear pathology reports is guiding for the treatment of cervicovaginal disease. Clinicians may consider the advantage of the CPS technique in detecting cervicovaginal infectious agents.

Ethics Committee Approval: Retrospective study.

Peer-review: Externally peer-reviewed.

Authorship Contributions: Concept: B.H.B., I.K., S.G.E.; Design: I.K.; Data Collection or Processing: B.H.B., I.K.; Analysis or Interpretation: B.H.B., I.K., S.G.E.; Literature Search: B.H.B., I.K., S.G.E.; Writing: I.K.

Conflict of Interest: None declared.

Financial Disclosure: The authors declared that this study received no financial support. 


\section{References}

1. Takei H, Ruiz B, Hicks J. Comparion of Conventional Pap Smears and a Liquid-Based Thin-Layer Preparation. Am J Clin Pathol 2006;125:855-9. [CrossRef]

2. Al-Awadhi R, Al-Shaheen A, Al-Juwaiser A, George SS, Sharma $P$, Kapila K. Prevalence of Infectious Organisms Observed in Cervical Smears Between 1997-2014 at Mubarak Al-Kabeer Hospital, Kuwait. Sultan Qaboos Univ Med J 2018;18:e324-8.
3. Cheung AN, Szeto EF, Leung BS, Khoo US, Ng AW. Liquid-based cytology and conventional cervical smears: a comparison study in an Asian screening population. Cancer 2003;99:3315. [CrossRef]

4. Yeoh GP, Chan KW, Lauder I, Lam MB. Evaluation of the ThinPrep Papanicolaou test in clinical practice: 6-month study of 16,541 cases with histological correlation in 220 cases. Hong Kong Med J 1999;5:233-9. 